If carers are getting Housing Benefit or Community Charge Benefit, they should still claim for Invalid Care Allowance. The local authority takes the Carer Premium into account when they work out how much Housing Benefit or Community Charge Benefit is payable. However, the carer must tell the office that pays Income Support, Housing Benefit or Community Charge Benefit that they get Invalid Care Allowance in order to get the extra help.

\section{How to claim}

There are two ways to claim.

(a) A form can be picked up from a local post office or local Social Security Office; the telephone number and address are in the telephone book under Social Security or Benefits Agency.

(b) One can contact the invalid Care Allowance Unit directly, their address is:

ICA Unit

Palatine House

Lancaster Road

Preston PR1 INS

Telephone: 0253856123

For further advice there is:

(a) The Citizens Advice Bureau. (b) Freeline Social Security 0800666 555. The phone call is free.

(c) The carer can get in touch with: The Carers' National Association 29 Chilworth Mews London W2 3RG

Telephone: 0717247776

\section{If the carer cannot get Invalid Care Allowance}

It is worth noting that if carers are looking after someone who gets AA, CAA or DLA at the middle or higher rate for help with personal care, but cannot get Invalid Care Allowance, they still may get help from the 'Home Responsibilities Protection Scheme'.

This is a scheme to make sure that people do not get less State Retirement Pension in the future just because they stay at home to look after someone. Carers should ask for form CF 411 Home Responsibilities Protection, which is available from any post office or Social Security Office.

The first article in this series was published in the June 1992 issue of the Psychiatric Bulletin and the second in the October 1992 issue.

\title{
The Merck, Sharp and Dohme Prize in Psychiatry
}

The winner of the 1992 Merck, Sharp and Dohme Prize in Psychiatry (East Anglia) is Dr Tony Rao, Senior House Officer at Fulbourn Hospital, Cambridge, with an entry on 'Suicide Risk in Dementia: An Under-Recognised Concept?'.

The Academic Department of Psychiatry announces that the competition has now been opened for the 1993 MSD Prize: first prize $£ 250$ and second prize $£ 80$. SHOs and registrars in psychiatry working in the East Anglian Region are eligible. For details, please contact: Ms Mary Coburn, PGME/ Medical Services Manager, Fulbourn Hospital, Cambridge CB1 5EF (telephone 0223 218673). 